\title{
The magnetic and electronic structure of vanadyl pyrophosphate from density functional theory $\dagger$
}

\author{
Mu-Jeng Cheng, Robert J. Nielsen, Jamil Tahir-Kheli and William A. Goddard III* \\ Received 3rd December 2010, Accepted 13th March 2011 \\ DOI: $10.1039 / \mathrm{c0cp02777d}$
}

We have studied the magnetic structure of the high symmetry vanadyl pyrophosphate $\left((\mathrm{VO})_{2} \mathrm{P}_{2} \mathrm{O}_{7}, \mathrm{VOPO}\right)$, focusing on the spin exchange couplings, using density functional theory (B3LYP) with the full three-dimensional periodicity. VOPO involves four distinct spin couplings: two larger couplings exist along the chain direction ( $a$-axis), which we predict to be antiferromagnetic, $J_{\mathrm{OPO}}=-156.8 \mathrm{~K}$ and $J_{\mathrm{O}}=-68.6 \mathrm{~K}$, and two weaker couplings appear along the $c$ (between two layers) and $b$ directions (between two chains in the same layer), which we calculate to be ferromagnetic, $J_{\text {layer }}=19.2 \mathrm{~K}$ and $J_{\text {chain }}=2.8 \mathrm{~K}$. Based on the local density of states and the response of spin couplings to varying the cell parameter $a$, we found that $J_{\mathrm{OPO}}$ originates from a super-exchange interaction through the bridging $-\mathrm{O}-\mathrm{P}-\mathrm{O}-$ unit. In contrast, $J_{\mathrm{O}}$ results from a direct overlap of $3 \mathrm{~d}_{x^{2}-y^{2}}$ orbitals on two vanadium atoms in the same $\mathrm{V}_{2} \mathrm{O}_{8}$ motif, making it very sensitive to structural fluctuations. Based on the variations in $\mathrm{V}-\mathrm{O}$ bond length as a function of strain along $a$, we found that the $\mathrm{V}-\mathrm{O}$ bonds of $\mathrm{V}-(\mathrm{OPO})_{2}-\mathrm{V}$ are covalent and rigid, whereas the bonds of $\mathrm{V}-(\mathrm{O})_{2}-\mathrm{V}$ are fragile and dative. These distinctions suggest that compression along the $a$-axis would have a dramatic impact on $J_{\mathrm{O}}$, changing the magnetic structure and spin gap of VOPO. This result also suggests that assuming $J_{\mathrm{O}}$ to be a constant over the range of 2-300 K whilst fitting couplings to the experimental magnetic susceptibility is an invalid method. Regarding its role as a catalyst, the bonding pattern suggests that $\mathrm{O}_{2}$ can penetrate beyond the top layers of the VOPO surface, converting multiple $\mathrm{V}$ atoms from the +4 to +5 oxidation state, which seems crucial to explain the deep oxidation of $n$-butane to maleic anhydride.

\section{Introduction}

Vanadium phosphorus oxide (VPO) is a most fascinating and unique catalyst system that selectively catalyzes the partial oxidation of $n$-butane $\left(\mathrm{C}_{4} \mathrm{H}_{10}\right)$ to maleic anhydride $\left(\mathrm{MA}, \mathrm{C}_{4} \mathrm{O}_{3} \mathrm{H}_{2}\right)$ (Scheme 1).

This very deep oxidation process involves 14 electrons, leading to $65 \%$ selectivity despite what must be a very complex and structure sensitive mechanism. ${ }^{1}$ Yet it is the only commercial application of alkane selective oxidation by a heterogeneous gas-solid catalytic process. ${ }^{1}$ Numerous studies have been directed toward understanding the reaction mechanism underlying this remarkable system, but no clear consensus has emerged. ${ }^{1}$ The most active phase has been identified as vanadyl pyrophosphate, (VO) ${ }_{2} \mathrm{P}_{2} \mathrm{O}_{7}$ (denoted VOPO). ${ }^{1}$

Materials and Process Simulation Center, California Institute of

Technology, Pasadena CA 91125, USA.

E-mail:wag@wag.caltech.edu; Tel:16263952731

$\dagger$ Electronic supplementary information (ESI) available: DFToptimized cell parameters and atomic coordinates of VOPO and spin configurations for least-square fitting are provided in ESI. See DOI: $10.1039 / \mathrm{c} 0 \mathrm{cp} 02777 \mathrm{~d}$
Of particular interest is the fact that each vanadium atom of VOPO is in the +4 oxidation state, leaving one unpaired electron in a $3 \mathrm{~d}$ orbital. This leads to a particularly complex antiferromagnetic coupling and this has stimulated numerous experimental ${ }^{2-9}$ and computational studies. ${ }^{10-14}$

The magnetic structure of VOPO was first thought to be a Heisenberg spin- $\frac{1}{2}$ ladder model. ${ }^{6,15}$ However, Johnston et al. found that the powder magnetic susceptibility data of VOPO can be equally accurately fitted by the spin $-\frac{1}{2}$ alternating antiferromagnetic (AFM) Heisenberg chain model. ${ }^{6}$ More recently, Garrett et al. used the inelastic neutron scattering experiments to demonstrate that VOPO has intrachain spin couplings that are one order of magnitude larger than the interchain couplings. Thus, the magnetic structure can be well described as an alternating AFM spin-chain along the $a$ axis

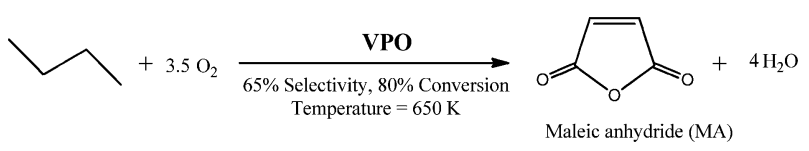

Scheme 1 Selective oxidation of $n$-butane to maleic anhydride catalyzed by VPO. 


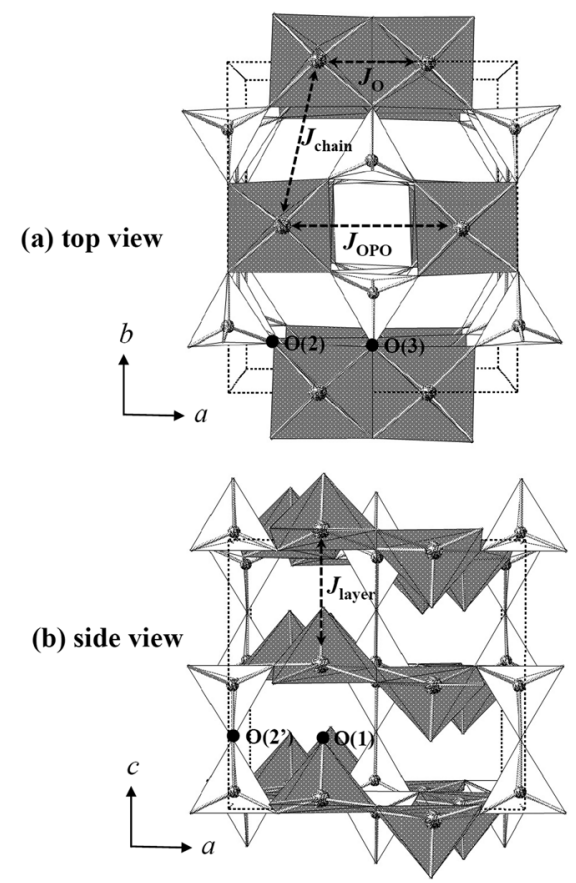

Fig. 1 Polyhedral views of the crystal structure of VOPO and the schematic description of the spin exchange couplings. The gray polyhedron represents the $\mathrm{V}_{2} \mathrm{O}_{8}$ motif $\left(\mathrm{V}_{2} \mathrm{O}_{2}\right.$ ring $)$ while the white represents $\mathrm{P}_{2} \mathrm{O}_{7}$. The bulk structure has four distinct types of oxygen: (a) $\mathrm{O}(1)$ : forming a formal vanadyl double bond $(\mathrm{V}=\mathrm{O})$ to a single vanadium atom; (b) $\mathrm{O}(2)$ : the doubly coordinated oxygen forming two covalent single bonds $(\mathrm{V}-\mathrm{O}-\mathrm{P}-\mathrm{O}-\mathrm{V})$ that bridges the $\mathrm{VO}_{5}$ square pyramid and the $\mathrm{PO}_{4}$ tetrahedron along the $a$ axis; (c) $\mathrm{O}\left(2^{\prime}\right)$ : the other doubly coordinated oxygen forming partial covalent bonds to the two phosphorus atoms (P-O-P) along the $c$ axis; (d) $\mathrm{O}(3)$ : the triply coordinated oxygen bridging the two $\mathrm{VO}_{5}$ square pyramids to form the $\mathrm{V}_{2} \mathrm{O}_{8}$ motif. This type of oxygen forms $\mathrm{P}=\mathrm{O}$ double bonds to the $\mathrm{P}$, making only dative (donor-acceptor) bonds to the $\mathrm{V}$.

(with alternating spin exchange couplings $J_{\mathrm{OPO}}$ and $J_{\mathrm{O}}$ in the chain, Fig. 1) rather than by the ladder model. ${ }^{5}$ However, they could not explain the two spin gaps observed. ${ }^{5}$ Yamauchi et al. ${ }^{9}$ and Kikuchi et al. ${ }^{7}$ used NMR and high-field magnetization studies to confirm the alternating AFM chain interpretation and, in addition, to explain the two spin gaps as due to two different types of alternating chains (with only slight geometric differences). Subsequently, Saito et al. showed that at high pressure VOPO leads to a higher symmetry structure with only a single chain (one spin gap). ${ }^{8}$ From magnetic susceptibility measurements over the range $2-300 \mathrm{~K}$, they were able to deduce the $J_{\mathrm{OPO}}$ and $J_{\mathrm{O}}$ spin couplings using the assumption that both couplings were temperature-independent. ${ }^{8}$

There have been no previous computational attempts to evaluate the spin couplings of VOPO using the threedimensional crystal structure. However, QM calculations (B3LYP) have been reported for cluster models of VOPO. ${ }^{10-14}$ Petit et al. ${ }^{13}$ confirmed that the interchain couplings are one order of magnitude smaller than the intrachain couplings, consistent with the experimental conclusions that the magnetic structure of VOPO can be described as a spin $-\frac{1}{2}$ alternating AFM Heisenberg model. Their cluster models lead to $J_{\mathrm{OPO}}=-144 \mathrm{~K}$ or $-200 \mathrm{~K}$ and $J_{\mathrm{O}}=-105 \mathrm{~K}$.
Despite numerous studies on this system, the nature of the spin couplings that result in this unique magnetic structure remain uncertain. To better understand this issue, we carried out quantum mechanical (QM) studies of the orthorhombic, high pressure, high symmetry form of VOPO (space group: $P n a b)^{8}$ using three-dimensional periodic conditions.

We show that the $J_{\mathrm{O}}$ coupling is exceptionally dependent on the lattice parameter $a$, changing from -190.2 (antiferromagnetic) to $+31.2 \mathrm{~K}$ (ferromagnetic) with a change in the lattice parameter of $8 \%$. This results from the dative nature of the bonds in the $\mathrm{V}-\mathrm{O}-\mathrm{V}$ bridges. We propose that this bonding pattern plays a crucial role in the catalysis of $n$-butane oxidation. ${ }^{16}$

\section{Computational details}

All calculations used the B3LYP ${ }^{17-19}$ flavor of densityfunctional theory (DFT), with full three-dimensional periodicity. B3LYP has been shown to predict band gaps of inorganic oxides accurately, since it provides a better description of the localization of the d orbitals. ${ }^{20,21}$ Thus, B3LYP is expected to yield spin exchange couplings more accurately than nonhybrid methods, such as PBE and PW91. ${ }^{22,23}$ To describe the various antiferromagnetic and ferromagnetic spin couplings, we used spin-unrestricted and spatially unrestricted B3LYP (U-B3LYP, as implemented in the CRYSTAL06 code).

The reciprocal space was sampled with a shrink factor of 3 to define the $k$-point net (eight $k$-points in the irreducible Brillouin zone), which we found is sufficiently dense to provide good numerical accuracy. Each atom is described by an all-electron, Gaussian-type basis set: $86-411 \mathrm{~d} 31 \mathrm{G}$ for $\mathrm{V}, 24,25$ $85-21 \mathrm{~d} 1 \mathrm{G}$ for $\mathrm{P},{ }^{26}$ and $8-411 \mathrm{~d} 11 \mathrm{G}$ for $\mathrm{O},{ }^{27}$ all of which have double zeta plus polarization quality.

We used the primitive orthorhombic $a \times b \times c$ unit cell to study the electronic structures of VOPO (Fig. 1), which has 52 atoms per unit cell. To study the magnetic structure, we used a doubled $2 a \times b \times c$ supercell in order to allow the description of more complex spin configurations. We define the $x$-axis along the $a$-direction, the $y$-axis along the $b$-direction, and the $z$-axis along the $c$-direction.

We use DFT broken-symmetry wavefunctions to access the diagonal elements of the Heisenberg spin Hamiltonian. The calculated $J$ can be compared to the experimental number obtained based on the same Hamiltonian. This has been proved and verified in recent theoretical works. ${ }^{28,29}$ The Hamiltonian and algorithm used to determine spin couplings from unrestricted DFT energies is detailed in the appendix.

Since DFT may lead to small systematic errors in determining the equilibrium geometries that might induce systematic changes in the spin exchange coupling, which are very sensitive to geometry, we used the experimental cell parameters and atomic positions ${ }^{8}$ in all studies of the VOPO magnetic and electronic structure, unless specifically stated otherwise.

In addition, we carried out a full optimization of the VOPO bulk structure, including atomic positions and cell parameters under orthorhombic symmetry. This was done to assess the adequacy of the DFT description of this system. The optimized cell parameters are $a=7.669 \AA, b=9.617 \AA$, and $c=8.453 \AA$ at $0 \mathrm{~K}$, in a good agreement with experimental values at $300 \mathrm{~K}$ 
$(a=7.571 \AA, b=9.536 \AA$, and $c=8.362 \AA) .{ }^{8}$ In addition, we found that the calculated atomic coordinates (Table S1 in ESI $\dagger$ ) deviate from the experimental values with an RMS of only $0.07 \AA$. This indicates that the U-B3LYP functional and basis set combination provides a good description of VOPO, making it suitable for this study.

\section{Results and discussion}

\subsection{Electronic structure of VOPO}

We first studied the electronic structure of this material to determine the bond character, band structure and density of states. ${ }^{30}$ From the spin density analysis of the unrestricted DFT wavefunction, we found that the singlet ground state (an equal number of up and down spins) has one unpaired spin on each vanadium atom, as expected for $\mathrm{V}^{\mathrm{IV}}$ (the Mulliken population analysis leads to net spin densities of $=+1.11$ or $-1.11 \mathrm{e}^{-}$). This shows that the unpaired electrons are highly localized on each vanadium. We also found that the optimum spin coupling configuration is antiferromagnetic (AFM) along the chains ( $a$ axis), while the coupling is ferromagnetic (FM) between the chains along the $b$ and $c$ axis, consistent with experimental results. ${ }^{5,8}$

The Mulliken charge of VOPO shows that the most nucleophilic site is $\mathrm{O}(3)\left(-1.08 \mathrm{e}^{-}\right)$, followed by $\mathrm{O}(2)\left(-0.93 \mathrm{e}^{-}\right)$, $\mathrm{O}\left(2^{\prime}\right)\left(-0.91 \mathrm{e}^{-}\right)$, and then $\mathrm{O}(1)\left(-0.76 \mathrm{e}^{-}\right)$. The larger magnitude for $\mathrm{O}(3)$ is expected, since it has a double bond to phosphorus and two electropositive vanadium neighbors. This suggests that $\mathrm{O}(3)$ may play a role in the physical adsorption of the $n$-butane substrate through electrostatic interactions with the positive hydrogen atoms (Mulliken charges of $\sim+0.10 \mathrm{e}^{-}$).

Our calculated band structure of VOPO (Fig. 2) shows a direct band gap of $3.61 \mathrm{eV}$ at the $\mathrm{X}$ point of the Brillouin zone, which is qualitatively consistent with the experimental

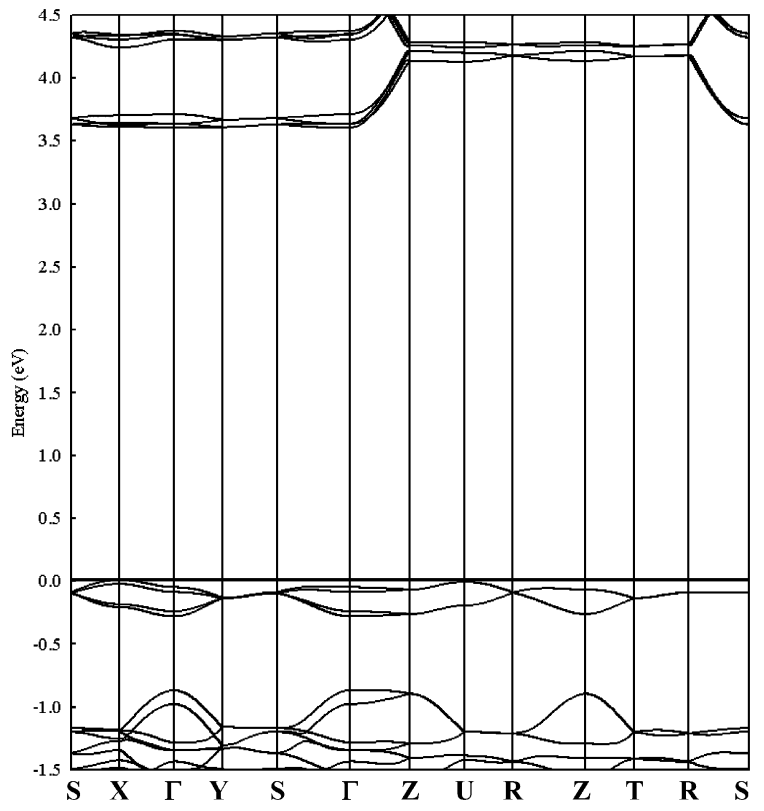

Fig. 2 The calculated band structure of VOPO. The solid horizontal line marks the top of the valence bands, taken as zero energy. observation. The color is observed to be transparent green ${ }^{8}$ suggesting a band gap larger than $1.8 \mathrm{eV}$. In contrast, DFT calculations at the PW91 and LDA level give much smaller band gaps of 0.81 and $0.48 \mathrm{eV}$, respectively. It is well known that these GGA- and LDA-type functionals lead to band gaps of oxides that are too small by $2-3 \mathrm{eV}$ due to the inability to account for the self-exchange hole. This causes excess delocalization of the electron density in strongly correlated materials containing transition metal or rare-earth metal ions with partially filled $3 \mathrm{~d}$ or $4 \mathrm{f}$ orbitals. ${ }^{31}$

We found that the HOMO bands (with energy of $0-0.3 \mathrm{eV}$ below the solid horizontal line) have band widths of only $\sim 0.2 \mathrm{eV}$, which indicates highly localized atomic-like orbitals. In order to obtain a further insight into these frontier orbitals, we projected the density of states (DOS) onto the various atoms (Fig. 3 and Fig. S1 in the ESI $\dagger$ ) and orbitals (Fig. S2 $\dagger$ ). The result shows that the HOMO orbitals are essentially pure $\mathrm{V} 3 \mathrm{~d}_{x^{2}}-y^{2}$ with slight delocalization onto the $\mathrm{O}(2) 2 \mathrm{p}_{x, y}$.

(a) $\mathrm{V}$ atoms

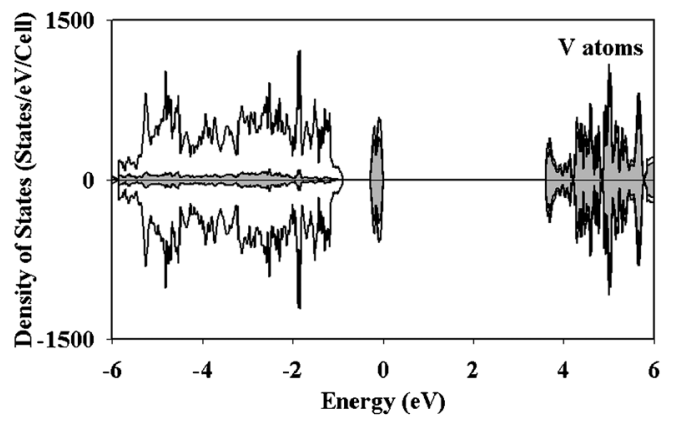

(b) $\mathrm{O}(1)$ atoms

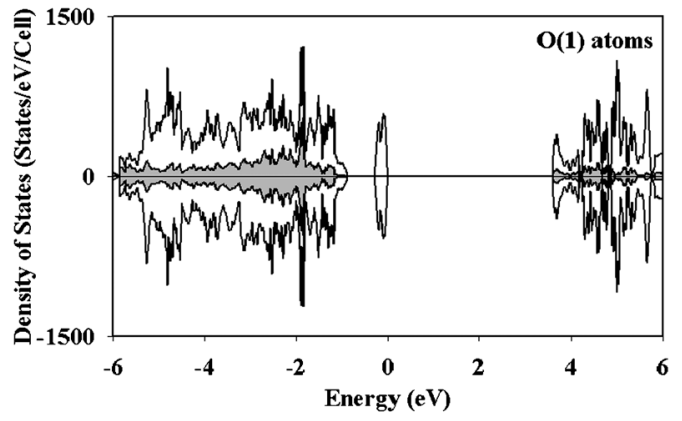

(c) $\mathrm{O}(2)$ atoms

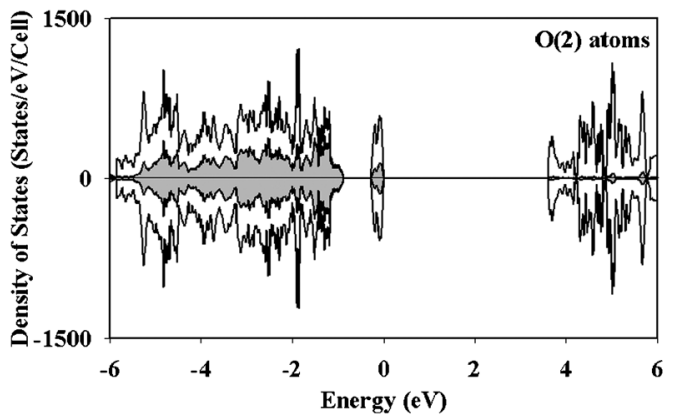

Fig. 3 The projected density of states (shaded) for $\mathrm{V}, \mathrm{O}(1)$, and $\mathrm{O}(2)$ atoms superimposed on the total density of states (unshaded) for VOPO. The zero energy is placed at the top of the valence bands. 
(a)

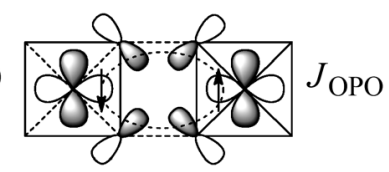

(b)

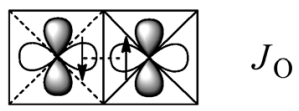

Scheme 2 Orbital Interactions responsible for the spin couplings $J_{\mathrm{OPO}}$ and $J_{\mathrm{O}}$.

From analyzing the eigenvectors, we found that there is an antibonding coupling between $\mathrm{V} 3 \mathrm{~d}_{x^{2}}-y^{2}$ and $\mathrm{O}(2) 2 \mathrm{p}_{x, y}$ (Scheme 2(a)). It is the overlap between the two $\mathrm{O}(2)$ on the same $\mathrm{P}(\mathrm{V}-\mathrm{O}(2)-\mathrm{P}-\mathrm{O}(2)-\mathrm{V})$ that is responsible for the superexchange coupling resulting in the AFM coupling. A similar description was proposed by Koo et al. ${ }^{10}$ based on reduced cluster models.

On the other hand, the LUMO orbitals (bands with energy of $3.6-4.5 \mathrm{eV}$ above the solid horizontal line) have widths of $\sim 0.8 \mathrm{eV}$ and a maximum energetic dispersion along the $\Gamma \rightarrow Z$ direction, implying a strong coupling of the antibonding orbitals on one $\mathrm{V}=\mathrm{O}$ with those of the adjacent $\mathrm{V}=\mathrm{O}$ along the $c$ axis. Those orbitals are comprised of $\mathrm{V} 3 \mathrm{~d}_{x z, y z}$ and $\mathrm{O}(1)$ $2 \mathrm{p}_{x, y}$, with $\mathrm{V} 3 \mathrm{~d}_{x z, y z}-\mathrm{O}(1) 2 \mathrm{p}_{x, y} \pi^{*}$ character. One may expect that $\mathrm{V}=\mathrm{O}(1)$ could play a role in $n$-butane $\mathrm{C}-\mathrm{H}$ activation through interactions between $\mathrm{V}=\mathrm{O}(1) \pi^{*}$ and $\mathrm{C}-\mathrm{H} \sigma$ bonds. Indeed, Robert et al. have proposed that $\mathrm{V}=\mathrm{O}$ is involved in $\mathrm{C}-\mathrm{H}$ bond cleavage. ${ }^{32}$ In contrast, $\mathrm{O}\left(2^{\prime}\right), \mathrm{O}(3)$ and $\mathrm{P}$ have no significant contributions to either the HOMO or LUMO.

\subsection{Magnetic structure of VOPO}

Having elucidated the electronic structure of VOPO, we further studied its magnetic structure, focusing on the spin exchange couplings between nearest-neighbor pairs of vanadium atoms. Four different types of spin couplings are present in VOPO (Fig. 1):

$J_{\mathrm{O}}$ : the spin coupling between a pair of vanadium atoms within the $\mathrm{V}_{2} \mathrm{O}_{8}$ motif connected by two $-\mathrm{O}(3)(=\mathrm{P})$ - units.

$J_{\mathrm{OPO}}$ : the spin coupling between a pair of vanadium atoms linked by two $-\mathrm{O}(2)-\mathrm{P}-\mathrm{O}(2)-$ units.

$J_{\text {chain }}$ : the spin coupling between vanadium atoms in two adjacent chains (interchain interaction), connected through $-\mathrm{O}(3)-\mathrm{P}-\mathrm{O}(2)-$.

$J_{\text {layer }}$ : the spin coupling between two vanadium atoms in two adjacent layers (interlayer interaction), connected indirectly through $\mathrm{O}(1)$.

The ground state from the UDFT calculations (denoted as Conf. 1, Table 1) is taken as the reference point.

We first determined $J_{\text {chain }}$ by flipping all of the spins in the second chain on both layers (Conf. 2), so that the interchain interactions all become AFM instead of FM. The energy of Conf. 2 increases by only $10.0 \mathrm{~K}$, suggesting that interchain interactions are $\mathrm{FM}$ with $J_{\text {chain }} \approx+0.6 \mathrm{~K}$.

Next, we evaluated the interlayer interaction by flipping all of the spins in the second layer, so that the interlayer interaction is AFM instead of FM (Conf. 3). We found that the energy increases by $333.6 \mathrm{~K}$, indicating that interlayer interactions are FM with $J_{\text {layer }} \approx+21 \mathrm{~K}$. The stronger interlayer
Table 1 Relative energies (in $\mathrm{K}$ ) for various spin configurations of VOPO. The energy components for each spin configuration are based on the formula derived in the appendix

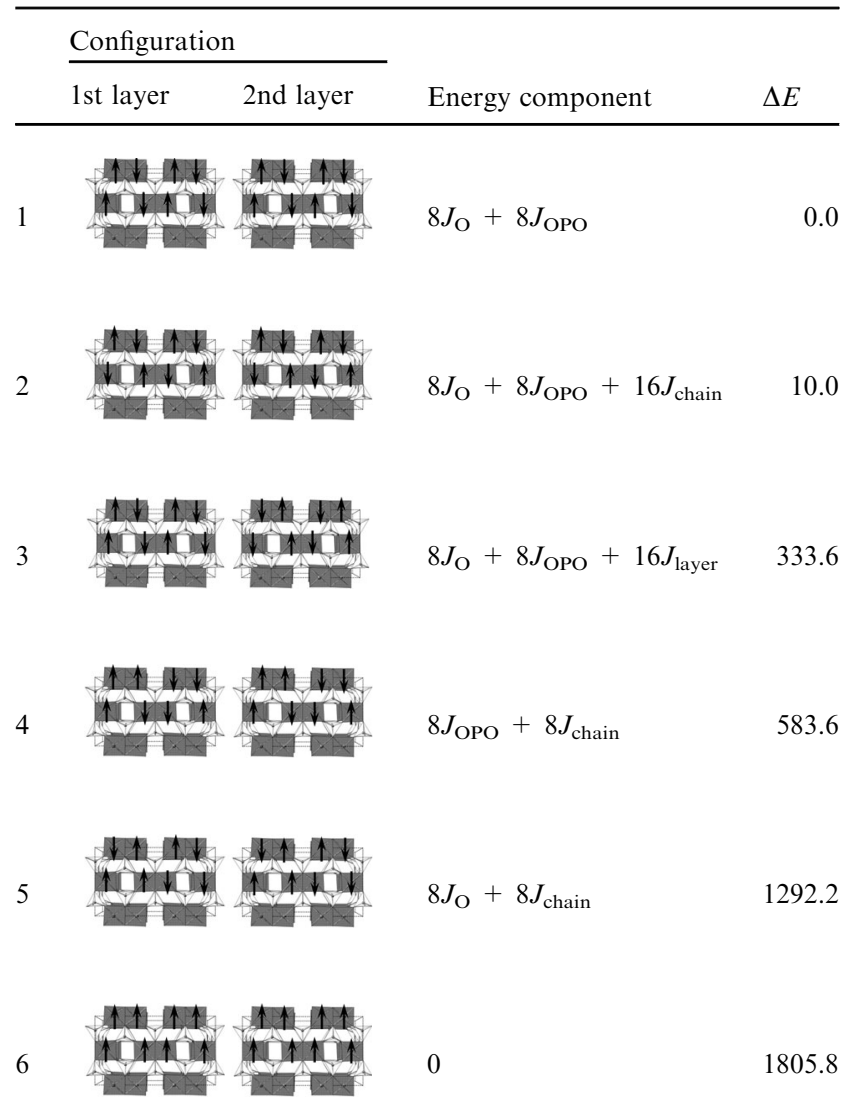

Table 2 A comparison between the experimental and theoretically predicted values of $J$ parameters in the high symmetry, high pressure form of VOPO (unit: K)

\begin{tabular}{lllll}
\hline & \multicolumn{2}{l}{ Intrachain } & Interlayer & \begin{tabular}{l} 
Interchain \\
\cline { 2 - 4 } Reference
\end{tabular} \\
\cline { 2 - 5 }$J_{\mathrm{O}}$ & $J_{\mathrm{OPO}}$ & $J_{\text {layer }}$ & $J_{\text {chain }}$ \\
\hline This work & -68.6 & -156.8 & 19.2 & 2.8 \\
Ref. 8 (exp) & -114.5 & -131.6 & - & - \\
Ref. 13 (theory) & -105 & $-144,-200$ & 37 & 6
\end{tabular}

${ }^{a}$ Ref. 8 assumes that both $J_{\mathrm{O}}$ and $J_{\mathrm{OPO}}$ are constants over the range 2-300 K.

coupling compared to the interchain coupling is due to the closeness of each $\mathrm{V}=\mathrm{O}(1)$ to the $\mathrm{V}=\mathrm{O}(1)$ of the next layer and the small direct charge transfer.

We then investigated the two types of intrachain interaction ( $J_{\mathrm{O}}$ first and then $J_{\mathrm{OPO}}$ ). Conf. 4 has FM coupling for $J_{\mathrm{O}}$, but AFM coupling for $J_{\mathrm{OPO}}$, leading to an energy $583.6 \mathrm{~K}$ higher than the ground state. This arises from eliminating $8 J_{\mathrm{O}}$ and adding $8 J_{\text {chain }}$. On the other hand, Conf. 5 introduces $F M$ coupling in $J_{\mathrm{OPO}}$, while $J_{\mathrm{O}}$ and the interlayer interaction remain the same as the ground state. This leads to an energy 1292.2 $\mathrm{K}$ higher than the ground state, due to the removal of $8 J_{\mathrm{OPO}}$ and adding $8 J_{\text {chain }}$. Thus comparing the energy between Conf. 4 and 5 , we deduce $J_{\mathrm{OPO}}-J_{\mathrm{O}} \approx-89 \mathrm{~K}$ indicating much stronger AFM coupling for $J_{\mathrm{OPO}}$ than $J_{\mathrm{O}}$. This shows that 


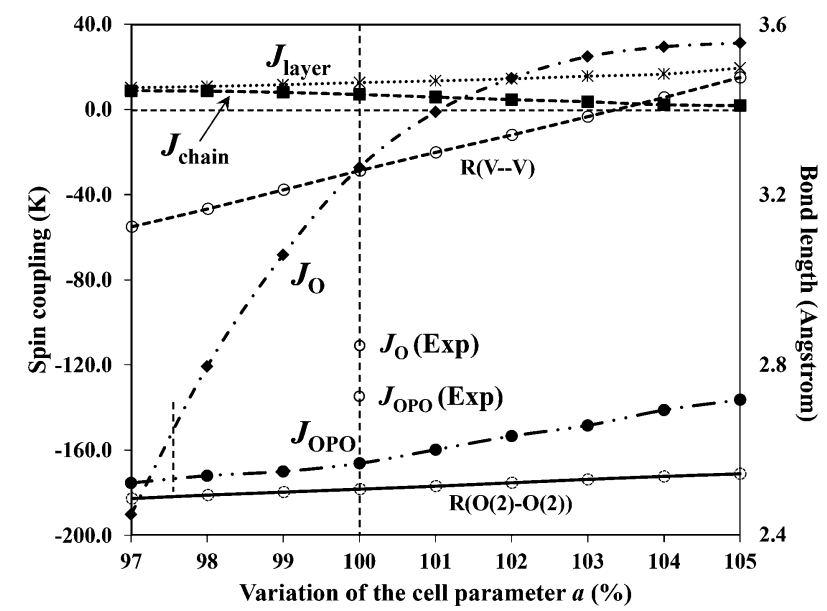

Fig. 4 The dependence of the spin couplings on the cell parameter $a$. At the experimental $a$, we calculated $J_{\mathrm{O}} / J_{\mathrm{OPO}}$ to be 0.44 . However, compressing $a$ by just $2.5 \%$ (indicated by the short black vertical line) leads to $J_{\mathrm{O}} / J_{\mathrm{OPO}}=0.89$, similar to the experimental value of 0.87 . The horizontal dashed line marks $J=0$.

$-\mathrm{O}(2)-\mathrm{P}-\mathrm{O}(2)-$ provides a better exchange pathway than $-\mathrm{O}(3)$ - in VOPO.

In order to calculate the most accurate spin exchange couplings, we calculated 10 additional different spin configurations (Table S2, ESI $\dagger$ ) for VOPO. Then, we did a least-square fitting to these energies to obtain the magnitude for each of the four $J$ parameters defined above.

These results lead to spin couplings of $J_{\mathrm{OPO}}=-156.8 \mathrm{~K}$ and $J_{\mathrm{O}}=-68.6 \mathrm{~K}$ (Table 2), indicating that the dominant magnetic structure of VOPO can be captured by a onedimensional Heisenberg alternating chain model, as suggested by inelastic neutron scattering experiments. ${ }^{5}$ This also indicates that the best interaction pathway is through the $-\mathrm{O}(2)-\mathrm{P}-\mathrm{O}(2)$ - bridge with a $\mathrm{V}-\mathrm{V}$ separation of $5.22 \AA$, rather than $-\mathrm{O}(3)$ - with a $\mathrm{V}-\mathrm{V}$ distance of $3.23 \AA$, which also agrees with the NMR experiments. ${ }^{7}$

The interlayer interaction is $J_{\text {layer }}=19.2 \mathrm{~K}(\mathrm{FM})$ with a $\mathrm{V}-\mathrm{V}$ separation of $3.79 \AA$, while the interchain coupling is $J_{\text {chain }}=2.8 \mathrm{~K}(\mathrm{FM})$ with a $\mathrm{V}-\mathrm{V}$ separation of $4.87 \AA$. The smaller couplings compared to the intrachain interactions suggest that direct, ferromagnetic exchange interactions dominate over antiferromagnetic ones.

Comparing with the experimental results, ${ }^{8}$ we found that our calculated $J_{\mathrm{OPO}}$ deviates from the experimental value $\left(J_{\mathrm{OPO}}=-131.6 \mathrm{~K}\right)$ by $19.1 \%$. This is probably partly due to the limitations of DFT, but it may arise from the use of a one-dimensional model to extract $J$ from the experiments, ignoring the interlayer $(19.2 \mathrm{~K})$ and interchain couplings $(2.8 \mathrm{~K})$, which may influence the intrachain couplings. Our $J_{\mathrm{OPO}}$ is consistent with one $(-144 \mathrm{~K})$ of a number calculated by Petit et al., ${ }^{13}$ but not with the other number $(-200 \mathrm{~K})$ based on a different cluster model.

Of most concern is the large discrepancy (45.9\% deviation) between the theory and experiment for $J_{\mathrm{O}}$, with the experimental value $(-114.5 \mathrm{~K})^{8}$ being nearly twice as high as the calculated value $(-68.6 \mathrm{~K})$. As we show in the following sections, this may be due to weak dative $\mathrm{V}-\mathrm{O}(3)$ bonds in the $\mathrm{V}_{2} \mathrm{O}_{8}$ motif, making $J_{\mathrm{O}}$, a direct spin exchange coupling and therefore extremely sensitive to structural fluctuations. The exponential dependence of $J_{\mathrm{O}}$ on the lattice parameter $a$ (or $R(\mathrm{~V}-\mathrm{V})$ ) invalidates the assumption that both $J_{\mathrm{OPO}}$ and $J_{\mathrm{O}}$ are constants over the range $2-300 \mathrm{~K}$ made by Saito et al. ${ }^{8}$ in fitting the couplings to their magnetic susceptibility versus temperature data. Assuming from the equipartition theorem that the $\frac{1}{2} \mathrm{k} T$ of potential energy is stored in the $\mathrm{V}-(\mathrm{O})_{2}-\mathrm{V}$ coordinate, the dependence of the total energy on $a$ (Fig. S3 $\dagger$ ) suggests fluctuations of $\sim 3 \%$ at room temperature, which could change the average value of $J_{\mathrm{O}}$ by more than $50 \%$. This may be responsible for the large discrepancy between our theoretically predicted and Saito's experimentally fitted couplings.

\subsection{Influence of the cell parameter $a$ in the magnetic structure}

In order to elucidate the nature of the spin exchange couplings in VOPO, we examined the dependence of $J$ on the cell parameter $a$. To do so, we compressed $a$ from the experimental value by $3 \%$ and expanded it by $5 \%$, in each case optimizing all of the atomic positions using the FM spin configuration. Here, we solved for the six spin configurations (Table S $3 \dagger$ ) and carried out least-squares fitting to obtain the spin couplings as a function of $a$. For the experimental structure, the use of just six spin configurations instead of 16 gives $J_{\mathrm{OPO}}=-158.5 \mathrm{~K}$, $J_{\mathrm{O}}=-69.9 \mathrm{~K}, J_{\text {layer }}=17.8 \mathrm{~K}$, and $J_{\text {chain }}=8.4 \mathrm{~K}$, which are quite similar to those obtained based on 16 configurations $\left(J_{\mathrm{OPO}}=-156.8 \mathrm{~K}, J_{\mathrm{O}}=-68.6 \mathrm{~K}, J_{\text {layer }}=19.2 \mathrm{~K}\right.$, and $J_{\text {chain }}=2.8 \mathrm{~K}$ ).

Indeed the cell parameter $a$ has a dramatic impact on $J_{\mathrm{O}}$ (Fig. 4). At $3 \%$, compression $(R(\mathrm{~V}-\mathrm{V})=3.13 \AA), J_{\mathrm{O}}$ is $\mathrm{AFM}$ with a value of $-190.2 \mathrm{~K}$, which is even larger in magnitude than $J_{\mathrm{OPO}}=-175.4 \mathrm{~K}$. As $a$ expands, the magnitude of $J_{\mathrm{O}}$ decreases rapidly, changing sign to $\mathrm{FM}\left(J_{\mathrm{O}}=+31.2 \mathrm{~K}\right)$ at $5 \%$ expansion $(R(\mathrm{~V}-\mathrm{V})=3.48 \AA)$. The large variation of $J_{\mathrm{O}}$ with the cell parameter $a$ (or the $\mathrm{V}-\mathrm{V}$ bond distance) and the insignificant contribution of $\mathrm{O}(3)$ to the HOMO indicate that $J_{\mathrm{O}}$ results from the overlap of $3 \mathrm{~d}_{x^{2}}-y^{2}$ orbitals of a pair of vanadium atoms in the same $\mathrm{V}_{2} \mathrm{O}_{8}$ motif, a direct throughspace exchange coupling (Scheme 2(b)). The same type of exchange mechanism is also responsible for the AFM ground state of the hydroxo- and alkoxo-bridged dinuclear oxovanadium(IV) compounds. ${ }^{33}$

In contrast, $J_{\mathrm{OPO}}, J_{\text {layer }}$, and $J_{\text {chain }}$ are relatively insensitive to the cell parameter $a$. $J_{\mathrm{OPO}}$ varies from -175.4 to $-136.4 \mathrm{~K}$, $J_{\text {layer }}$ varies from 10.2 to $19.4 \mathrm{~K}$ and $J_{\text {chain }}$ varies from 8.8 to $1.8 \mathrm{~K}$, none of which change sign.

Our discovery that $J_{\mathrm{O}}$ is highly sensitive to the cell parameter $a$ shows that the magnetic structure of VOPO depends on $a$. Thus for the $3 \%$ to $0 \%$ compression, the system is best described by a spin- $\frac{1}{2}$ Heisenberg AFM alternating chain model, while for the $1 \%$ to $3 \%$ expansion it is best described by a spin-dimer model. These results also suggest that the spin gap of the spin- $\frac{1}{2}$ Heisenberg alternating chain, which is a function of $J_{\mathrm{O}}$ and $J_{\mathrm{OPO}},{ }^{34}$ depends very sensitively on the cell parameter $a$. Consequently, we suggest that the spin gap and magnetic structure of VOPO depends sensitively on pressure. 


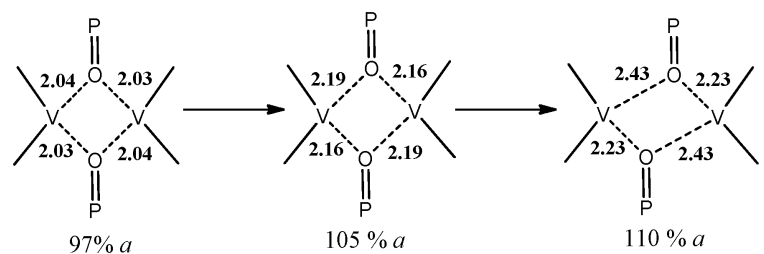

Scheme 3 Variation of $\mathrm{V}-\mathrm{O}$ bond lengths with cell parameter $a$.

Analyzing the structural parameters with different $a$ parameters, we found that $R(\mathrm{~V}-\mathrm{O}(3))$ increases from 2.04 and $2.03 \AA$ to 2.19 and $2.16 \AA$ (6.9\% variation), respectively, as the a lattice parameter increases from $97 \%$ to $105 \%$. Further expanding $a$ to $110 \%$ increases the $\mathrm{V}-\mathrm{O}(3)$ bond lengths to 2.43 and $2.23 \AA$. At this point, we found that the $\mathrm{V}_{2} \mathrm{O}_{8}$ cluster distorts such that one $\mathrm{P}=\mathrm{O}(3)$ group makes a donor-acceptor bond to one $\mathrm{V}$, while the other $\mathrm{P}=\mathrm{O}(3)$ group makes a donor-acceptor bond to the other V (Scheme 3).

In contrast, $\mathrm{V}-\mathrm{O}(2)$ increases from 1.92 to $1.98 \AA$ (only $3.0 \%$ variation), as the $a$ lattice parameter is changed from $97 \%$ to $105 \%$. Further expanding to $110 \%$ leads to the $\mathrm{V}-\mathrm{O}(2)$ bond length of $1.98 \AA$, the same as that of the $5 \%$ expansion. Based on this asymmetric relaxation of atomic positions, we see that $\mathrm{V}-\mathrm{O}(2)$ has a rigid covalent single bond, while $\mathrm{V}-\mathrm{O}(3)$ has a fragile dative bond. The valence bond structure of VOPO is shown in Scheme 4.

To illustrate this, we built a cluster model (the inset of Fig. 5) containing this $J_{\mathrm{O}}$ spin coupling, ${ }^{35}$ in which the phenomenon is reproduced. The spin coupling is AFM with a $J_{\mathrm{O}}=-274.7 \mathrm{~K}$ when the $\mathrm{V}-\mathrm{V}$ distance is fixed at $3.1 \AA$. This coupling is weakened as $R(\mathrm{~V}-\mathrm{V})$ increases, and finally becomes FM with a value of $+8.4 \mathrm{~K}$ at $R(\mathrm{~V}-\mathrm{V})=3.6 \AA$. This result is similar to that based on the three-dimensional bulk model, where $J_{\mathrm{O}}$ changes from $-190.2 \mathrm{~K}$ at $R(\mathrm{~V}-\mathrm{V})=3.13 \AA$ to $+31.2 \mathrm{~K}$ at $R(\mathrm{~V}-\mathrm{V})=3.48 \AA$. Importantly, we found the energy cost required to totally dissociate this cluster into two fragments is only $9.2 \mathrm{kcal} \mathrm{mol}^{-1}$, suggesting that the $\mathrm{V}-\mathrm{O}(3)$ binding energy is only $4.6 \mathrm{kcal} \mathrm{mol}^{-1}$.

\subsection{Implication for the catalytic reaction}

TAP (temporal analysis of products) reactor experiments ${ }^{36}$ showed that with a sufficient dose of oxygen on the VOPO surface prior to exposure to $n$-butane, subsequent exposure to butane leads to selectively fully oxidized maleic anhydride, without intermediates being released. This shows that the reaction is localized and that all seven oxygen required for

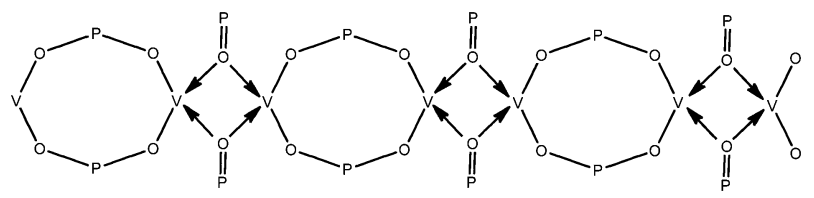

Scheme 4 The valence bond description of the bonding along the chain. The $\mathrm{P}$ has three $\mathrm{P}-\mathrm{O}$ bonds not shown plus a double bond to $\mathrm{O}(3)$, so that $\mathrm{O}(3)$ makes only dative bonds (donor-acceptor or Lewis base-acid bonds) to the two vanadium atoms. This weak bonding leads to no significant super-exchange so that the direct V $3 \mathrm{~d}_{x^{2}}-y^{2}-\mathrm{V}$ $3 \mathrm{~d}_{x^{2}-y^{2}}$ overlap dominates, leading to an exponential dependence of $J_{\mathrm{O}}$ on the distance.

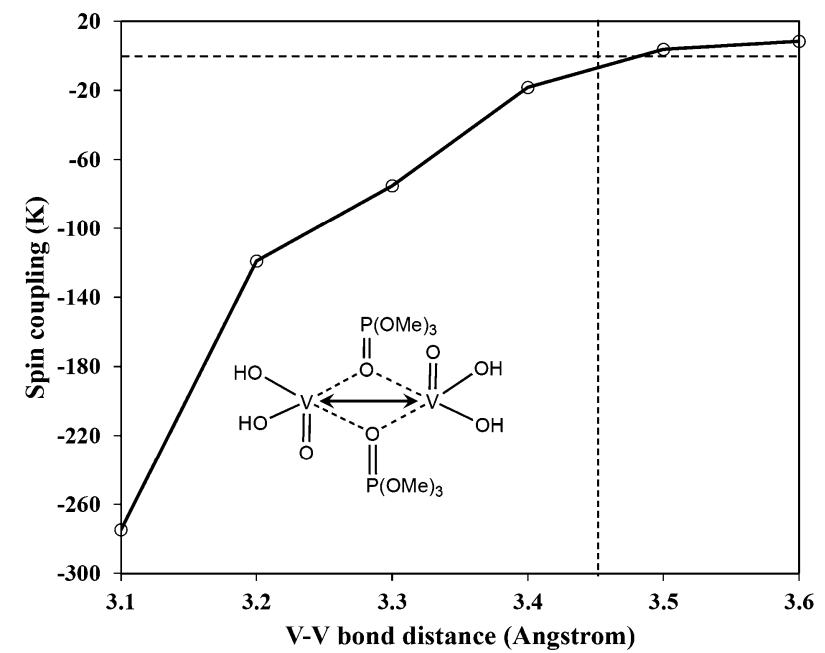

Fig. 5 The dependence of the spin coupling on the $\mathrm{V}-\mathrm{V}$ distance in the $\mathrm{V}_{2} \mathrm{O}(3)_{2}$ cluster model. The horizontal dashed line marks $J=0$, and the vertical dashed line marks the equilibrium at the $\mathrm{V}-\mathrm{V}$ distance (3.47 $\AA$ )

completing the reaction are somehow stored near the surface and are accessible to the substrate. Indeed, it has been shown by ${ }^{31} \mathrm{P}$ NMR that in the working VOPO catalyst, a small portion of $\mathrm{V}^{+4}$ has been oxidized to $\mathrm{V}^{+5} \cdot 37,38$ Moreover, Coulston et al. showed that a small amount of $\mathrm{V}^{+5}$ is crucial for a successful catalyst, based on in situ X-ray absorption spectroscopy studies. ${ }^{16}$

We consider that the observation that there is essentially no interaction between $\mathrm{O}(3)$ and vanadium is important, because it suggests that gas phase $\mathrm{O}_{2}$ can penetrate to subsurface layers, while oxidizing the vanadium atoms from the +4 to +5 oxidation state to store sufficient $\mathrm{O}$ atoms for the full oxidation. That is, under thermal expansion or chemical reactions, the $\mathrm{V}_{2} \mathrm{O}(3)_{2}$ four-membered rings may open up forming a channel, so that gaseous $\mathrm{O}_{2}$ molecules can go inside VOPO and oxide multiple layers.

\section{Conclusions}

From DFT calculations, we found that the magnetic structure of VOPO can be described in terms of four different types of spin couplings in VOPO: $J_{\mathrm{OPO}}=-156.8 \mathrm{~K}, J_{\mathrm{O}}=-68.6 \mathrm{~K}$, $J_{\text {layer }}=19.2 \mathrm{~K}$, and $J_{\text {chain }}=2.8 \mathrm{~K}$. This confirms that the magnetic structure of VOPO is best described by a spin- $\frac{1}{2}$ Heisenberg AFM alternating chain model, consistent with experiments by inelastic neutron scattering ${ }^{5}$ and NMR. ${ }^{7,9}$

By varying the cell parameter $a$ and analyzing the responses of spin exchange couplings and structural parameters, we found that $J_{\mathrm{OPO}}$ and $J_{\mathrm{O}}$ result from two very different exchange mechanisms: $J_{\mathrm{OPO}}$ originates from super-exchange interactions through the $-\mathrm{O}(2)-\mathrm{P}-\mathrm{O}(2)-\operatorname{link}$, whereas $J_{\mathrm{O}}$ results from a direct overlap of the $3 \mathrm{~d}_{x^{2}}-y^{2}$ orbitals of two vanadium atoms in the same $\mathrm{V}_{2} \mathrm{O}_{8}$ motif and, therefore, it is very sensitive to structural fluctuations. Moreover, we also found that the $\mathrm{V}-\mathrm{O}$ bonds of $\mathrm{V}-(\mathrm{O}(2) \mathrm{PO}(2))_{2}-\mathrm{V}$ are covalent and rigid, whereas the bonds of $\mathrm{V}-(\mathrm{O}(3))_{2}-\mathrm{V}$ are fragile and dative. These results suggest that pressure would dramatically 
change the magnetic structure and spin gap of VOPO, which should be observable experimentally. This also suggests that assuming $J_{\mathrm{O}}$ to be constant over the range of $2 \sim 300 \mathrm{~K}$ in fitting couplings to the experimental magnetic susceptibility is an invalid method.

Regarding catalysis, the bonding pattern suggests that under thermal expansion or chemical reaction, the $\mathrm{V}_{2} \mathrm{O}(3)_{2}$ four-membered rings may open up forming a channel, so that multiple $\mathrm{O}_{2}$ molecules can be stored inside the VOPO by reacting with vanadium atoms beneath the surface.

\section{Appendix}

The interaction between two magnetic ions can be described by a Heisenberg spin Hamiltonian

$$
\hat{H}^{\text {spin }}=-J \hat{S}_{1} \cdot \hat{S}_{2}
$$

where $J$ is the spin exchange parameter and $\hat{S}_{1}$ and $\hat{S}_{2}$ are the spin angular momentum operators for two adjacent magnetic sites. The system favors parallel spins (FM) if $J$ is positive and antiparallel (AFM) if $J$ is negative.

Considering just two spins, we can write the total spin operator as $\hat{S}=\hat{S}_{1}+\hat{S}_{2}$ leading to $\hat{S}^{2}=\hat{S}_{1}{ }^{2}+\hat{S}_{2}{ }^{2}+2 \hat{S}_{1}$. $\hat{S}_{2}$. Hence eqn (1) can be rewritten as

$$
\hat{H}^{\text {spin }}=-\frac{J}{2}\left(\hat{S}^{2}-\hat{S}_{1}^{2}-\hat{S}_{2}^{2}\right)
$$

Assuming just one unpaired electron on each site, we take $\hat{S}_{1}=\hat{S}_{2}=0.5$, leading to $E_{\mathrm{T}}=-0.25 \mathrm{~J}$ for the triplet state and $E_{\mathrm{S}}=0.75 \mathrm{~J}$ for the singlet. Consequently, the $J$ parameter can be obtained by comparing the energy difference between the singlet and triplet states

$$
J=E_{\mathrm{S}}-E_{\mathrm{T}}
$$

However, the current generation of DFT methods do not allow the multiple determinants needed to describe pure singlet states. For two electrons, the singlet state requires two determinants (the simple GVB wavefunction). Thus, for two electrons, our calculation on the AFM singlet state leads to the wave function:

$$
\psi_{\mathrm{S}^{\prime}}=\frac{1}{\sqrt{2}}[a(1) \alpha(1) b(2) \beta(2)-b(1) \beta(1) a(2) \alpha(2)],
$$

where $a$ and $b$ are localized, singly occupied orbitals. The wave functions of the correct singlet and triplet states are:

$$
\begin{aligned}
& \psi_{\mathrm{S}}=\frac{1}{2 \sqrt{1+S_{a b}^{2}}}[a(1) b(2)+b(1) a(2)][\alpha(1) \beta(2)-\beta(1) \alpha(2)] \\
& \psi_{\mathrm{T}}=\frac{1}{2 \sqrt{1-S_{a b}^{2}}}[a(1) b(2)-b(1) a(2)][\alpha(1) \beta(2)+\beta(1) \alpha(2)] .
\end{aligned}
$$

where $S_{a b}$ is the overlap of two singly occupied magnetic orbitals and we have assumed that the orbitals $a$ and $b$ are the same for singlet and triplet. Writing $\psi_{\mathrm{S}^{\prime}}$ as a linear combination of $\psi_{\mathrm{S}}$ and $\psi_{\mathrm{T}}$ :

$$
\psi_{\mathrm{S}^{\prime}}=\sqrt{\frac{1+S_{a b}^{2}}{2}} \psi_{\mathrm{S}}+\sqrt{\frac{1-S_{a b}^{2}}{2}} \psi_{\mathrm{T}}
$$

the energy becomes

$$
E_{\mathrm{S}^{\prime}}=\left(\frac{1+S_{a b}^{2}}{2}\right) E_{\mathrm{S}}+\left(\frac{1-S_{a b}^{2}}{2}\right) E_{\mathrm{T}}=\left(\frac{1+2 S_{a b}^{2}}{4}\right) J
$$

This leads to a relation between $J, E_{\mathrm{S}^{\prime}}$, and $E_{\mathrm{T}}$ :

$$
J=\frac{2\left(E_{\mathrm{S}^{\prime}}-E_{\mathrm{T}}\right)}{1+S_{a b}^{2}}
$$

In the VOPO systems, $S_{a b}$ is approximately zero, since the unpaired electrons are highly localized at vanadium atoms. As a result, eqn (4) becomes:

$$
J=2 \times\left(E_{\mathrm{S}^{\prime}}-E_{\mathrm{T}}\right) .
$$

This formula was first proposed by Noodleman ${ }^{39}$ in 1981 and has been successfully applied to numerous magnetic systems. We use eqn (5) to obtain the $J$ parameters in VOPO.

\section{Acknowledgements}

This work was supported by the Center for Catalytic Hydrocarbon Functionalization, an Energy Frontier Research Center, DOE DE-SC0001298.

\section{Notes and references}

1 B. K. Hodnett, Heterogeneous Catalytic Oxidation, John Wiley \& Sons Ltd, New York, NY, 2000.

2 M. Azuma, T. Saito, Y. Fujishiro, Z. Hiroi, M. Takano, F. Izumi, T. Kamiyama, T. Ikeda, Y. Narumi and K. Kindo, Phys. Rev. B: Condens. Matter, 1999, 60, 10145-10149.

3 E. Dagotto and T. M. Rice, Science, 1996, 271, 618-623.

4 A. W. Garrett, S. E. Nagler, T. Barnes and B. C. Sales, J. Appl. Phys., 1997, 81, 3968-3970.

5 A. W. Garrett, S. E. Nagler, D. A. Tennant, B. C. Sales and T. Barnes, Phys. Rev. Lett., 1997, 79, 745-748.

6 D. C. Johnston, J. W. Johnson, D. P. Goshorn and A. J. Jacobson, Phys. Rev. B, 1987, 35, 219-222.

7 J. Kikuchi, K. Motoya, T. Yamauchi and Y. Ueda, Phys. Rev. B: Condens. Matter, 1999, 60, 6731-6739.

8 T. Saito, T. Terashima, M. Azuma, M. Takano, T. Goto, H. Ohta, W. Utsumi, P. Bordet and D. C. Johnston, J. Solid State Chem., 2000, 153, 124-131.

9 T. Yamauchi, Y. Narumi, J. Kikuchi, Y. Ueda, K. Tatani, T. C. Kobayashi, K. Kindo and K. Motoya, Phys. Rev. Lett., 1999, 83, 3729-3732.

10 H. J. Koo and M. H. Whangbo, Inorg. Chem., 2000, 39, 3599-3604.

11 M. L. Lawson Daku, S. Borshch, V. Robert and B. Bigot, Phys. Rev. B: Condens. Matter, 2001, 63, 174439.

12 H. J. Koo, M. H. Whangbo, P. D. VerNooy, C. C. Torardi and W. J. Marshall, Inorg. Chem., 2002, 41, 4664-4672.

13 S. Petit, S. A. Borshch and V. Robert, J. Am. Chem. Soc., 2002, 124, 1744-1749.

14 S. Petit, S. A. Borshch and V. Robert, J. Solid State Chem., 2003, 170, 237-246.

15 R. S. Eccleston, T. Barnes, J. Brody and J. W. Johnson, Phys. Rev. Lett., 1994, 73, 2626-2629.

16 G. W. Coulston, S. R. Bare, H. Kung, K. Birkeland, G. K. Bethke, R. Harlow, N. Herron and P. L. Lee, Science, 1997, 275, 191-193.

17 A. D. Becke, Phys. Rev. A: At., Mol., Opt. Phys., 1988, 38, 3098-3100.

18 A. D. Becke, J. Chem. Phys., 1993, 98, 5648-5652.

19 C. T. Lee, W. T. Yang and R. G. Parr, Phys. Rev. B, 1988, 37, 785-789.

20 J. Muscat, A. Wander and N. M. Harrison, Chem. Phys. Lett., 2001, 342, 397-401

21 J. K. Perry, J. Tahir-Kheli and W. A. Goddard, Phys. Rev. B: Condens. Matter, 2001, 63, 144510. 
22 D. Munoz, N. M. Harrison and F. Illas, Phys. Rev. B: Condens. Matter Mater. Phys., 2004, 69, 085115.

23 R. L. Martin and F. Illas, Phys. Rev. Lett., 1997, 79, 1539-1542.

24 W. C. Mackrodt, N. M. Harrison, V. R. Saunders, N. L. Allan, M. D. Towler, E. Apra and R. Dovesi, Philos. Mag. A, 1993, 68, 653-666.

25 E. Ruiz, M. Llunell and P. Alemany, J. Solid State Chem., 2003, 176, 400-411.

26 C. M. Zicovich-Wilson, A. Bert, C. Roetti, R. Dovesi and V. R. Saunders, J. Chem. Phys., 2002, 116, 1120-1127.

27 L. Valenzano, F. J. Torres, D. Klaus, F. Pascale, C. M. ZicovichWilson and R. Dovesi, Z. Phys. Chem., 2006, 220, 893-912.

28 D. D. Dai and M. H. Whangbo, J. Chem. Phys., 2003, 118, 29-39.

29 I. D. R. Moreira and F. Illas, Phys. Rev. B: Condens. Matter, 1997, 55, 4129-4137.

30 DFT fully optimized VOPO leads to the same Mulliken populations, band structure, and density of states found using the experimental structure.
31 V. I. Anisimov, A. I. Poteryaev, M. A. Korotin, A. O. Anokhin and G. Kotliar, J. Phys.: Condens. Matter, 1997, 9, 7359-7367.

32 V. Robert, S. A. Borshch and B. Bigot, J. Mol. Catal. A: Chem., 1997, 119, 327-333.

33 A. Rodriguez-Fortea, P. Alemany, S. Alvarez and E. Ruiz, Eur. J. Inorg. Chem., 2004, 143-153.

34 J. C. Bonner and M. E. Fisher, Phys. Rev., 1964, 135, A640.

35 For the finite cluster model, all calculations were carried out with Jaguar 7.0 using the B3LYP hybrid-density functional. For the geometry optimizations, we used the Los Alamos effective core potential (ECP) with the double- $\zeta$ contraction of valence functions for vanadium and phosphorus atoms and $6-31 \mathrm{G}^{* *}$ for the others.

36 J. T. Gleaves, J. R. Ebner and T. C. Kuechler, Catal. Rev., 1988, 30, 49-116.

37 J. Li, M. E. Lashier, G. L. Schrader and B. C. Gerstein, Appl. Catal., 1991, 73, 83-95.

38 M. T. Sananes, A. Tuel and J. C. Volta, J. Catal., 1994, 145, 251-255.

39 L. Noodleman, J. Chem. Phys., 1981, 74, 5737-5743. 